Review Article

\title{
Hypertension May Reduce the Infection Risk but Increase the Severity of COVID-19: Based on the Current Data in China
}

\author{
Bo Li, ${ }^{1,2}$ Lu Zeng, ${ }^{3}$ Nengjun Sun, ${ }^{4}$ Yunhe Zhao, ${ }^{1,2}$ Faming Zhao, ${ }^{5}$ Hongjun Bian, ${ }^{6}$ Wei Yi, ${ }^{7}$ \\ Jing Yang, ${ }^{8}$ Bin Li $\mathbb{D}^{9,10}$ and Guohai Su $\mathbb{D}^{9,10}$ \\ ${ }^{1}$ Department of Cardiology, Shandong University Zibo Central Hospital, No. 10, South Shanghai Road, Zibo 255000, China \\ ${ }^{2}$ Department of Cardiology, Zibo Hospital Affiliated to Shandong First Medical University, Zibo, Shandong 255000, China \\ ${ }^{3}$ Weifang Medical University, No. 288, Shengli East Street, Weifang City, Shandong 261000, China \\ ${ }^{4}$ Department of Medical Administration, Shandong University Central Hospital of Zibo, No. 54, Gong Qing Tuan Xi Road, \\ Zibo 255036, China \\ ${ }^{5}$ Department of Infectious Diseases, No. 4, Hospital of Zibo, No. 210, Shan Quan Road, Zibo 255000, China \\ ${ }^{6}$ Departments of Emergency Medicine, Shandong Provincial Hospital Affiliated to Shandong First Medical University, \\ Jinan 250012, China \\ ${ }^{7}$ Department of Intelligent Manufacturing Teaching, Engineering Training Center, Shandong University, Jinan 250002, China \\ ${ }^{8}$ Binzhou Medical University, No. 346, Guanhai Road, Laishan District, Yantai 264003, China \\ ${ }^{9}$ Department of Cardiology, Central Hospital Affiliated to Shandong First Medical University, Jinan, Shandong,250013, China \\ ${ }^{10}$ Department of Cardiology, Jinan Central Hospital, Cheeloo College of Medicine, Shandong University, Jinan, \\ Shandong 250013, China
}

Correspondence should be addressed to Bin Li; qingquan0615@163.com and Guohai Su; gttstg@163.com

Received 2 July 2020; Accepted 3 November 2021; Published 20 December 2021

Academic Editor: Kwok Leung Ong

Copyright (C) 2021 Bo Li et al. This is an open access article distributed under the Creative Commons Attribution License, which permits unrestricted use, distribution, and reproduction in any medium, provided the original work is properly cited.

Increasing evidence has shown an unusual relationship between hypertension and COVID-19, which may not be as simple as previously thought. The purpose of our study was to determine the association of hypertension with the onset and development of COVID-19. A meta-analysis was performed to summarize the prevalence of hypertension in COVID-19 patients, as well as the usage of ACEIs/ARBs. Metaregression analyses were used to evaluate the association of hypertension with disease severity and mortality. PubMed and Google Scholar were searched for relevant studies. A total of 42 studies including 14138 patients were enrolled in the study. The proportion of hypertension in COVID-19 patients in China was $17.7 \%$ according to the enrolled studies, while it was $6.0 \%$ in a study containing 72314 confirmed cases, which are both much lower than in the general population. All of the data from the 11 provinces in China showed the same tendency. The proportions of hypertension were higher in severe/ICU patients and nonsurvivors than in nonsevere/ICU patients and survivors. The metaregression analyses suggested that both disease severity and risk of death were associated with the incidence of hypertension. A total of $27.6 \%$ of COVID-19 patients with hypertension received ACEI/ARB therapy. The proportion of deaths in COVID-19 patients with hypertension treated with ACEIs/ARBs was significantly lower than that in nonuse patients treated with ACEIs/ARBs. In conclusion, hypertension may reduce the infection risk of COVID-19 but increase the risk of developing worse clinical outcomes. The use of ACEIs/ARBs may benefit COVID-19 patients with hypertension. 


\section{Introduction}

With the publication of several studies concerning the epidemiological and clinical features of coronavirus disease 2019 (COVID-19), a growing amount of evidence is emerging about the relationship between cardiovascular complications and COVID-19. According to early published data in Wuhan, hypertension was associated with a high prevalence and an increased risk of morbidity and mortality.

In Wang's study, $31.2 \%$ of the 138 confirmed COVID-19 cases had hypertension [1]. Another study included 140 COVID-19 patients, of whom 42 patients $(30 \%)$ had hypertension [2]. The prevalence of hypertension in fatal patients was extremely high $(42.57 \%-60 \%)$ [3-5]. Thus, patients with hypertension seemed to be more likely to be infected with SARS-CoV-2 and face a greater risk of developing severe conditions $[6,7]$.

However, emerging evidence shows the reported prevalence of hypertension is gradually decreasing. In our previous study, the 6 earliest studies about COVID-19 were summarized, and the results suggested that the proportion of hypertension in patients with COVID-19 was $17.1 \%$ [8]. A study that included 1099 patients with confirmed COVID-19 reported that $14.9 \%$ of patients had hypertension comorbidities [9]. In another study, among 44672 individuals with confirmed COVID-19, 2683 patients (12.8\%) had hypertension [10]. Even more surprising was that in the largest study ever conducted in China thus far, including 72314 confirmed cases, only $6.0 \%$ of patients had hypertension [11]. Because the total number of infections in China was 84414 as of May 7th, the study was very representative of the total population. The incidence of $6.0 \%$ was much lower than that of the general population, which reported a $27.9 \%$ overall crude prevalence of hypertension according to the Summary of the 2018 report on cardiovascular diseases in China [12]. This information indicated that individuals with underlying hypertension seemed to be less susceptible to COVID-19. This discovery is very different from all others that have been made thus far. Thus, it is urgent to determine the relationship between hypertension and the virus.

Angiotensin-converting enzyme inhibitors (ACEIs) and angiotensin receptor blockers (ARBs) are the main antihypertensive drugs recommended in the current guidelines. However, angiotensin-converting enzyme 2 (ACE2) has been identified as a receptor for SARS-CoV-2, which infects humans by binding to ACE2 in host respiratory epithelial cells [13]. Therefore, the application of ACEIs/ARBs to control blood pressure in patients with COVID-19 complicated with hypertension has been a matter of intense debate $[14,15]$. Therefore, we will conduct an analysis on the incidence and prognosis of patients with COVID-19 complicated with hypertension, as well as the use of ACEIs/ARBs in infected patients and their impact on prognosis.

\section{Methods}

This meta-analysis was conducted following the Preferred Reporting Items for the Systematic Review and MetaAnalysis (PRISMA) statement [16].

2.1. Search Strategy and Selection Criteria. A literature search was conducted in MEDLINE via the PubMed database, Cochrane Library, Web of Science, EMBASE and EMBASE Classic databases, and Google Scholar from inception through April 23, 2020. The following terms (MeSH) were used: "2019nCoV," "COVID19 virus," "2019 novel coronavirus," "SARSCoV-2," and "hypertension." The included articles met the following eligibility criteria: (1) RCTs or non-RCTs; (2) patients were diagnosed with 2019 novel coronavirus infection; (3) sample size $\geq 10$ in each study population; and (4) comorbidities of hypertension and the outcome of hypertension were given. Studies not meeting these criteria, nonclinical studies, incompatible or repeated studies, case reports, and studies without complete data were excluded.

2.2. Data Management and Quality Assessment. Clinical data, including age, sex, prevalence of hypertension, clinical outcome, and interventions for hypertension, were collected from the identified studies. The primary outcomes were to analyse the prevalence of hypertension and the impact of hypertension on the severity of the disease and mortality. The secondary outcomes were to analyse the use of ACEIs/ ARBs in COVID-19 and the impact of ACEIs/ARBs on prognosis. Two reviewers assessed the eligibility of the included reports, extracted data with a standardized report form, and evaluated the quality of reports independently. All discrepancies were solved by discussion until a consensus was reached.

2.3. Statistical Analysis. The statistical analysis was performed using OpenMeta Analyst version 10.10 (https:// www.cebm.brown.edu/open_meta) and Review Manager (RevMan) software version 5.3. Forest plots were used to illustrate the prevalence of hypertension in 2019-nCoV infection from the selected studies as well as the impact of 2019-nCoV infection and therapeutic interventions on hypertension. Heterogeneity among studies was assessed by $I^{2}$ statistics. In the pooled analysis, a fixed-effects model was applied when the heterogeneity $I^{2}$ was less than $50 \%$; otherwise, a random-effects model was used $[17,18]$. Metaregression analyses were used to evaluate the associations between disease severity, risk of death, and incidence of hypertension. Cochrane Collaboration's tool was followed to assess the risk of bias.

\section{Results}

3.1. Selected Studies and Baseline Characteristics. We searched all the databases and websites using the search words described above, and 21052 total records were 
retrieved. Among the articles, 2987 records were excluded because of duplication. In addition, 17943 records were excluded because they were animal trials, letters, review articles, case reports, or repetitive content. The remaining 124 studies were carefully evaluated in detail. Finally, 93 studies were excluded because the participants of the trials did not meet the criteria we set. Then, 11 studies published in the preprint platform were added. Finally, a total of 42 articles were selected for this study (Table 1 and Figure 1).

3.2. Primary Outcomes. Forty-two studies, including 14138 COVID-19 patients with hypertension, were collected in the study. Meta-analysis of the identified studies showed that the prevalence of hypertension was $17.7 \%$ (95\% CI: 15.1-20.4\%) (Figure 2(a)). There was significant heterogeneity (Cochran's Q) in the estimates of hypertension among the identified studies with an $I^{2}$ index of $93.3 \%$. Next, 26 studies including 4458 patients outside of Hubei were also analysed, showing that the prevalence of hypertension was $14.3 \%$ (95\% CI: 13.3-15.3\%) (Figure 2(b)). Then, 14 studies including 6991 patients in Hubei Province were analysed, and the data showed that the prevalence of hypertension was $24.7 \%$ (95\% CI: 19.4-30.1\%) (Figure 2(c)).

Furthermore, we compared the prevalence of hypertension in COVID-19 patients with data from the China Hypertension Survey, 2012-2015 [19]. The results demonstrated the differences in all 11 provinces outside of Hubei whose data could be currently obtained (Figure 3 ) as follows: Beijing (35.9\% vs. $20.5 \%)$ [20, 21], Shanghai $(29.1 \%$ vs. $21.2 \%$ ) [22], Zhejiang (23.2\% vs. 13.4\%) [23-25], Hunan (15.6\% vs. $14.1 \%)$ [26-28], Guangdong ( $27.3 \%$ vs. $12.8 \%)$ [29], Chongqing (20.6\% vs. $7.8 \%$ ) [30], Sichuan (23.6\% vs. $5.88 \%$ ) [31], Hainan (20.3\% vs. $14.3 \%$ ) [32], Fujian (23.9\% vs. $14.5 \%)$ [33], Jiangsu (25.3\% vs. $7.0 \%)$ [34], and Tibet $(25.0 \%$ vs. $11.9 \%)$ [35] (Figure 3).

Then, the differences in the prevalence of hypertension between severe/intensive care unit (ICU) and nonsevere/ ICU patients and nonsurvivors and survivors were compared. There was significant statistical heterogeneity in the test results (severe/ICU and nonsevere/ICU patients: $I^{2}=82 \%$; nonsurvivors and survivors: $I^{2}=85 \%$ ). Therefore, a random-effects model was selected for further analysis. Studies with a total of 8004 patients showed that hypertension accounted for $28.0 \%$ of severe/ICU cases, but only $15.7 \%$ of nonsevere/ICU cases. The proportion of hypertension was significantly higher in severe/ICU patients than in nonsevere/ICU patients ( $\mathrm{RR}=1.91,95 \% \mathrm{CI}(1.48,2.48)$, $Z=4.90, P<0.00001$ ) (Figure 4(a)). For nonsurvivors and survivors with hypertension (with a total number of 2870 patients), the heterogeneity test showed that $I^{2}=85 \%$, and a random effects model was used. Hypertension was present in $52.1 \%$ of nonsurvivors but $20.8 \%$ of survivors. The results indicated a much higher proportion of hypertension in nonsurvivors than in survivors, with statistical significance $(\mathrm{RR}=1.71, \quad 95 \% \quad \mathrm{CI} \quad(1.19, \quad 2.46), \quad Z=2.91, \quad P=0.004)$ (Figure 4(b)). In metaregression analyses of the included studies, disease severity and risk of death were both associated with the incidence of hypertension $(\beta=0.010, P=$
0.032 and $\beta=0.009, P<0.001$, respectively) (Figures 5(a) and $5(\mathrm{~b}))$.

3.3. Secondary Outcomes. Finally, we collected data from COVID-19 patients with hypertension according to the use of ACEIs/ARBs. Four studies (including 1300 patients) showed that $27.6 \%$ (95\% CI: $15.8-39.4 \%$ ) of COVID-19 patients with hypertension received ACEI/ARB therapy (Figure 6(a)). Then, we compared the differences in the severe/ICU rate and mortality of COVID-19 patients with hypertension between the ACEI/ARB group and the nonACEI/ARB group. Meta-analysis showed that the proportion of deaths in COVID-19 patients with hypertension treated with ACEIs/ARBs was significantly lower than that in nonuse patients treated with ACEIs/ARBs $(\mathrm{RR}=0.41,95 \%$ CI $(0.21,0.81), Z=2.60, P=0.009$ ) (Figure 6(b)). The results also showed a slightly lower proportion of severe/ICU among patients treated with ACEIs/ARBs than in patients not treated with ACEIs/ARBs, but the difference was not statistically significant $(\mathrm{RR}=0.77,95 \%$ CI $(0.30,1.97)$, $Z=0.54, P=0.59)$ (Figure 6(c)).

\section{Discussion}

A growing number of studies on the epidemiological and clinical characteristics of COVID-19 suggest a relationship between cardiovascular complications and COVID-19. Hypertension is one of the most important complications in patients with COVID-19. According to earlier data in Wuhan, there was a high proportion of hypertension among confirmed cases, reaching more than $30 \%$. However, in a study covering 72314 confirmed cases, only $6.0 \%$ of patients had hypertension [11]. In the present study, we collected all of the published data involving provinces outside Hubei. We compared the prevalence of hypertension in COVID-19 patients with the data from the China Hypertension Survey, 2012-2015 [19]. The results demonstrated the differences in all 11 provinces whose data could be currently obtained. All of the incidences of hypertension in COVID-19 patients were lower than those in the general population. We do not believe this is just a coincidence. We hypothesized that hypertension might reduce the risk of SARS-CoV-2 infection.

However, in Hubei Province, the result was the opposite. One possible reason why the incidence of hypertension in COVID-19 patients was higher than that of the general population in Hubei was that many people with mild illnesses might have gone undiagnosed in the face of a collapsed health care system, which would reduce the accuracy of the data. However, when the healthcare system is healthy, milder and even asymptomatic patients can be diagnosed, which might result in a lower prevalence of hypertension outside of Hubei.

If the above hypothesis and interpretation can be accepted, there must be a premise as follows: the incidence of hypertension in mild patients is low, but it is high in severe patients. To test this premise, we analysed the prevalence of hypertension between severe and nonsevere patients and 
TABLe 1: Number, age, sex, and hypertension of patients of the included studies.

\begin{tabular}{|c|c|c|c|c|c|c|}
\hline References & Date & Number of patients & Area & Age & Sex (male, \%) & Hypertension (\%) \\
\hline Chaolin Huang & As of 2020.1 .2 & 41 & Wuhan & 49 & 73.2 & 14.6 \\
\hline Youbin Liu & 2020.1.10-2020.2.24 & 291 & Guangzhou & 48.1 & 45.7 & 18.6 \\
\hline Jie Li & 2020.1.22-2020.2.10 & 17 & Dazhou & 45 & 52.9 & 5.9 \\
\hline Guyi Wang & As of 2020.2 .20 & 242 & Changsha & 45 & 49.2 & 14.9 \\
\hline Yafei Wang & 2020.1.1-2020.2.10 & 110 & Wuhan & - & 43.6 & 20.9 \\
\hline Xiaowei Xu & 2020.1.10-2020.1.26 & 62 & 7 hospitals in Zhejiang & 41 & 56.5 & 8.1 \\
\hline Wanbo Zhu & 2020.1.24-2020.2.20 & 32 & Hefei & 46 & 46.9 & 21.9 \\
\hline Xu Chen & 2020.1.23-2020.2.14 & 291 & Changsha and Loudi & 46 & 49.8 & 13.4 \\
\hline Qingxian Cai & 2020.1.11-2020.2.6 & 298 & Shenzhen & 47 & 50 & 12.8 \\
\hline Dawei Wang & 2020.1.1-2020.1.28 & 138 & Wuhan & 56 & 54.3 & 31.2 \\
\hline W. Guan & $2019.12 .11-2020.1 .29$ & 1099 & 552 hospitals & 47 & 58.1 & 15.0 \\
\hline Fei Zhou & 2019.12.29-2020.1.31 & 191 & Wuhan & 56 & 62.3 & 30.4 \\
\hline Guoqing Qian & 2020.1.20-2020.2.11 & 91 & 7 hospitals in Zhejiang & 50 & 40.7 & 16.5 \\
\hline Guqin Zhang & 2020.1.2-2020.2.10 & 221 & Wuhan & 55 & 48.9 & 24.4 \\
\hline Pengfei Cui & $2020.1 .28-2020.2 .18$ & 35 & Wuhan & 61.5 & 0 & 34.3 \\
\hline Yu Lei & 2020.1.4-2020.2.28 & 67 & Daofu & 39.3 & 58.2 & 11.9 \\
\hline Lei Liu & $2020.1 .20-2020.2 .3$ & 51 & Chongqing & 45 & 62.7 & 7.8 \\
\hline Lei Wang & 2020.1.21-2020.2.5 & 18 & Zhengzhou & 39 & 55.6 & 27.8 \\
\hline Penghui Yang & 2019.12.27-2020.2.18 & 55 & Beijing & 44 & 60 & 20 \\
\hline Jinjin Zhang & 2020.1.16-2020.2.3 & 140 & Wuhan & 57 & 50.7 & 30 \\
\hline Jie Liu & 2020.1.16-2020.2.15 & 64 & Wuhan & 35 & 35.9 & 4.7 \\
\hline Min Cao & 2020.1.20-2020.2.15 & 198 & Shanghai & 50.1 & 51.0 & 21.2 \\
\hline Chengfeng Qiu & 2020.1.22-2020.2.12 & 104 & Huaihua & 43 & 47.1 & 14.4 \\
\hline Rui Huang & 2020.1.22-2020.2.10 & 221 & 10 hospitals in Jiangsu & 45 & 57.0 & 14.5 \\
\hline Zhijun Xie & 2020.1.22-2020.2.15 & 60 & Hangzhou & 45 & 45 & 15 \\
\hline Fengqin Zhang & 2020.1.30-2020.2.15 & 81 & Jinzhou & - & 55.6 & 13.6 \\
\hline Songqiao Liu & 2020.1.10-2020.2.18 & 620 & 24 hospitals in Jiangsu & 44.48 & 52.6 & 15.5 \\
\hline Weijie Guan & $2019.12 .11-2020.1 .31$ & 1590 & 575 hospitals & 48.9 & 57.3 & 17.0 \\
\hline Peng Zhang & 2019.12.31-2020.2.20 & 3430 & 9 hospitals in Hubei & - & 32.9 & 32.9 \\
\hline Juan Meng & 2020.1.11-2020.2.23 & 417 & Shenzhen & 64.5 & - & 12.2 \\
\hline Sijiao Wang & 2020.1.22-2020.2.16 & 165 & Fuzhou & 44 & 55.8 & 14.5 \\
\hline Shijiao Yan & $2020.1 .22-2020.3 .13$ & 168 & Haikou & 51 & 48.2 & 14.3 \\
\hline Xiufeng Jiang & $2020.1 .23-2020.2 .16$ & 55 & Wuxi & 45 & 49.1 & 30.9 \\
\hline Yu Shi & 2020.1.16-2020.2.17 & 487 & 5 hospitals in Zhejiang & 46 & 53.2 & 20.3 \\
\hline Huisi $\mathrm{He}$ & $2020.2 .8-2020.3 .16$ & 94 & Wuhan & 69.2 & 57.4 & 59.6 \\
\hline Caizheng Yu & 2020.1.14-2020.2.28 & 1663 & Wuhan & 64 & 50.4 & 20.9 \\
\hline Lin $\mathrm{Fu}$ & 2020.1.1-2020.1.30 & 200 & Wuhan & - & 49.5 & 50.5 \\
\hline Zhenhua Zeng & $2020.1 .5-2020.3 .8$ & 274 & Wuhan & 60 & 54.7 & 27.4 \\
\hline Xin Chen & 2020.2.11-2020.2.29 & 208 & Xiaogan & 50.5 & 51.4 & 19.7 \\
\hline Xingwei He & 2020.2.3-2020.2.24 & 54 & Wuhan & 68 & 63.0 & 44.4 \\
\hline $\mathrm{Bo} \mathrm{Hu}$ & $2020.1 .8-2020.2 .9$ & 50 & Wuhan & 62 & 68 & 36 \\
\hline Suxin Wan & 2020.1.23-2020.2.8 & 135 & Chongqing & 47 & 53.3 & 9.6 \\
\hline Yimei Yin & As of 2020.2 .15 & 112 & Wuhan & 66 & 68.8 & 43.8 \\
\hline Zhongbao Zuo & 2020.1.20-2020.2.28 & 70 & Hangzhou & 43 & 41.4 & 12.9 \\
\hline Wei Chen & 2020.1.19-2020.2.7 & 74 & Nanjing & 48.1 & 58.1 & 13.5 \\
\hline Jiaxi Chen & 2020.1.22-2020.2.26 & 137 & Taizhou & - & 52.6 & 12.4 \\
\hline \multirow[t]{2}{*}{ Wentao Xu } & $2020.1 .10-2020.2 .18$ & 87 & Suzhou & - & 52.9 & 6.9 \\
\hline & 2020.1.11-2020.2.5; & & Shenzhen & & & \\
\hline \multirow[t]{2}{*}{ Yingxia Liu } & 2020.1.12-2020.2.9; & 78 & Wuhan & - & - & 100 \\
\hline & 2019.12.27-2020.2.27 & & Beijing & & & \\
\hline Kun Wang & $2020.1 .7-2020.2 .11$ & 305 & Wuhan & 47.8 & 46.6 & 14.8 \\
\hline Fei Zhou & 2019.12.29-2020.1.31 & 191 & Wuhan & 56 & 62.3 & 30.4 \\
\hline
\end{tabular}

nonsurvivors and survivors. We found that patients with hypertension were more likely to develop severe cases or be nonsurvivors after SARS-CoV-2 infection. The total proportion of hypertension in severe/ICU patients was approximately 1.78 -fold that in nonsevere/ICU patients, while in nonsurvivors, it was 2.50-fold that in survivors, both of which showed significant differences. In metaregression analyses in patients with COVID-19, the disease severity and risk of death were both statistically associated with the incidence of hypertension.

Although the underlying mechanism is unknown, further investigation of the expression and activity of ACE2 is worthwhile. ACE2 is a carboxypeptidase that can hydrolyse Ang I to Ang-(1-9) and Ang II to Ang-(1-7) without being 
Flowchart of COVID-19

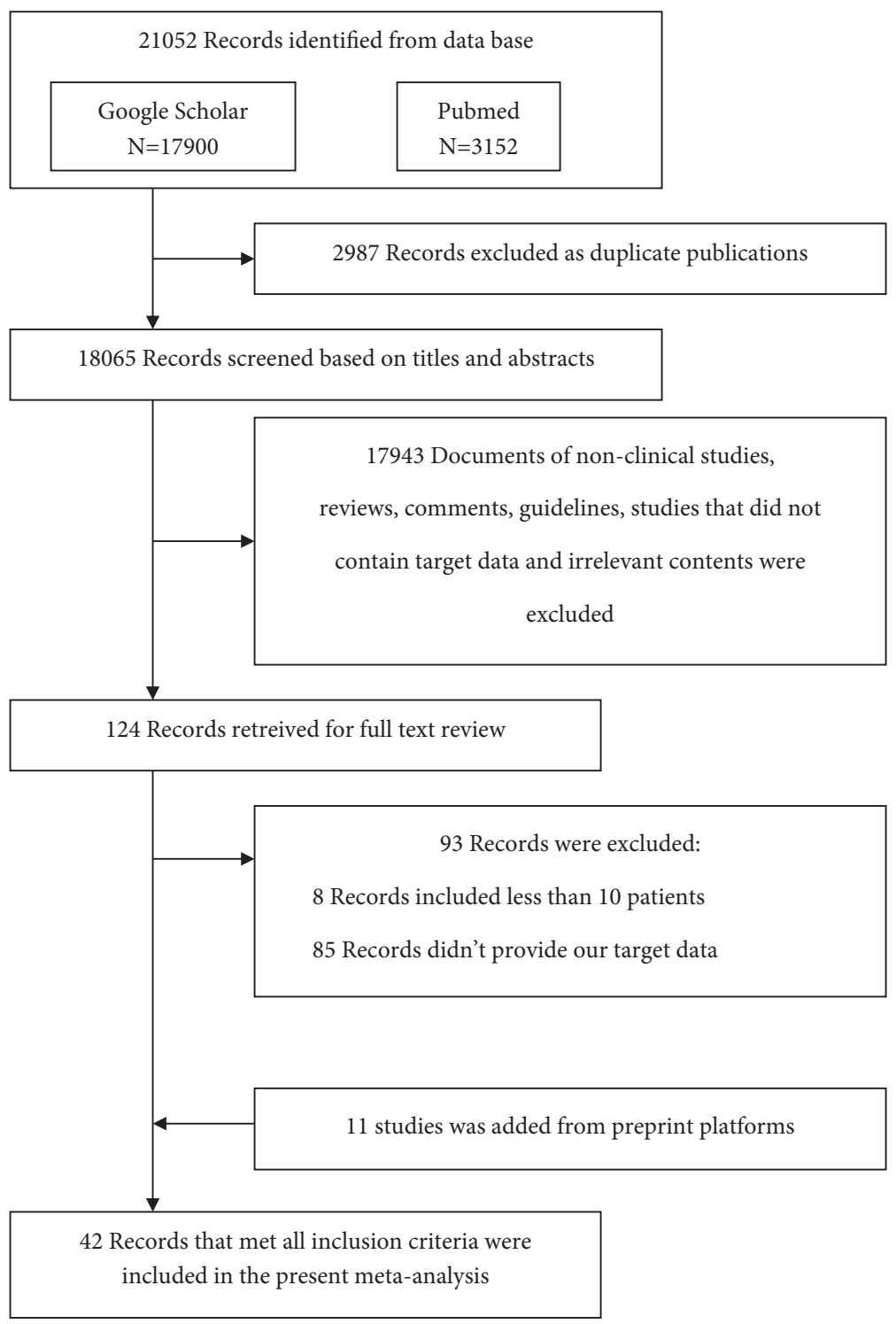

FIGURE 1: Flow diagram of the study selection process.

inhibited by selective ACE inhibitors. ACE2 has more favourable kinetics for the hydrolysis of Ang II than Ang I. [36] A previous study suggested that the ACE2 expression level was significantly downregulated in the kidneys of three hypertensive rat strains [37]. In a clinical study, hypertension status was also confirmed as an independent confounding determinant of the ACE to ACE2 ratio, leading to the relative downregulation of ACE2 [38]. SARS-CoV-2 binds to its target cells through ACE2, which is expressed in epithelial cells, type 2 pneumocytes, and macrophages in the lungs. Studies have speculated that high expression of ACE2 in patients with hypertension might facilitate SARS-CoV-2 entry into targeted cells in the respiratory system [13]. One of the main reasons why hypertension could reduce SARS$\mathrm{CoV}-2$ infection might be the loss of ACE2 in hypertensive subjects. ACE2 has a strong cardiovascular protective effect, which could also explain why patients with hypertension had a worse prognosis once they were infected with the virus. The downregulated expression of ACE2 after infection with SARS-CoV leads to excessive activation of the RAS [39], which activates peripheral sympathetic neuron release of catecholamine mediated by activation of multiple signalling pathways causing vasoconstriction and bronchial contraction [40]. It has been shown that the binding of coronavirus to ACE2 leads to the downregulation of ACE2, which in turn causes an ACE/ACE2 imbalance and an excessive production of angiotensin II by the related ACE enzyme. Excessive activation of the RAS also promotes inflammation, causes cytokine storms [41], generates ROS by activating the NADH/NADPH oxidase system [42], and induces cell apoptosis, thus promoting the progression of coronavirusrelated lung injury [43].

ACEIs and ARBs are the major RAS inhibitors commonly used in clinical practice and the main 
Studies

Chaolin Huang 2020

Youbin Liu 2020

Jie Li 2020

Guyi Wang 2020

Xiaowei Xu 2020

Wanbo Zhu 2020

Xu Chen 2020

Qingxian Cai 2020

Dawei Wang 2020

W. Guan 2020

Guoqing Qian 2020

Guqin Zhang 2020

Pengfei Cui 2020

Yu Lei 2020

Lei Liu 2020

Lei Wang 2020

Penghui Yang 2020

Jie Liu 2020

Jinjin Zhang 2020

Min Cao 2020

Chengfeng Qiu 2020

Rui Huang 2020

Zhijun Xie 2020

Fengqin Zhang 2020

Songqiao Liu 2020

Weijie Guan 2020

Peng Zhang 2020

Juan Meng 2020

Sijiao Wang 2020

Shijiao Yan 2020

Yu Shi 2020

Caizheng Yu 2020

Lin Fu 2020

Zhenhua Zeng 2020

Xin Chen 2020

Suxin Wan 2020

Zhongbao Zuo 2020

Wei Chen 2020

Jiaxi Chen 2020

Wentao Xu 2020

Kun Wang 2020

Fei Zhou 2020

Overall $\left(I^{\wedge} 2=93.3 \%, P<0.001\right)$
$0.177(0.151,0.204)$

Estimate (95\% C.I.)

$0.146(0.038,0.255)$

$0.186(0.141,0.230)$

$0.059(0.000,0.171)$

$0.081(0.013,0.148)$

$0.219(0.076,0.362)$

$0.134(0.095,0.173)$

$0.128(0.090,0.165)$

$0.312(0.234,0.389)$

$0.150(0.129,0.171)$

$0.165(0.089,0.241)$

$0.244(0.188,0.301)$

$0.343(0.186,0.500)$

$0.119(0.042,0.197)$

$0.078(0.005,0.152)$

$0.278(0.071,0.485)$

$0.200(0.094,0.306)$

$0.047(0.000,0.099)$

$0.300(0.224,0.376)$

$0.212(0.155,0.269)$

$0.144(0.077,0.212)$

$0.145(0.098,0.191)$

$0.150(0.060,0.240)$

$0.136(0.061,0.210)$

$0.155(0.126,0.183)$

$0.169(0.151,0.188)$

$0.329(0.313,0.345)$

$0.122(0.091,0.154)$

$0.145(0.092,0.199)$

$0.143(0.090,0.196)$

$0.203(0.168,0.239)$

$0.209(0.189,0.228)$

$0.505(0.436,0.574)$

$0.274(0.221,0.327)$

$0.197(0.143,0.251)$

$0.096(0.047,0.146)$

$0.129(0.050,0.207)$

$0.135(0.057,0.213)$

$0.124(0.069,0.179)$

$0.069(0.016,0.122)$

$0.148(0.108,0.187)$

$0.304(0.238,0.369)$
$0.149(0.104,0.194)$
Ev/Trt

$6 / 41$

$54 / 291$

$1 / 17$

$36 / 242$

$5 / 62$

$7 / 32$

$39 / 291$

$38 / 298$

$43 / 138$

$165 / 1099$

$15 / 91$

$54 / 221$

$12 / 35$

$8 / 67$

$4 / 51$

$5 / 18$

$11 / 55$

$3 / 64$

$42 / 140$

$42 / 198$

$15 / 104$

$32 / 221$

$9 / 60$

$11 / 81$

$96 / 620$

269/1590

$1128 / 3430$

$51 / 417$

$24 / 165$

24/168

99/487

$347 / 1663$

$101 / 200$

$75 / 274$

$41 / 208$

$13 / 135$

9/70

$10 / 74$

$17 / 137$

$6 / 87$

45/305

$58 / 191$

$3070 / 14138$
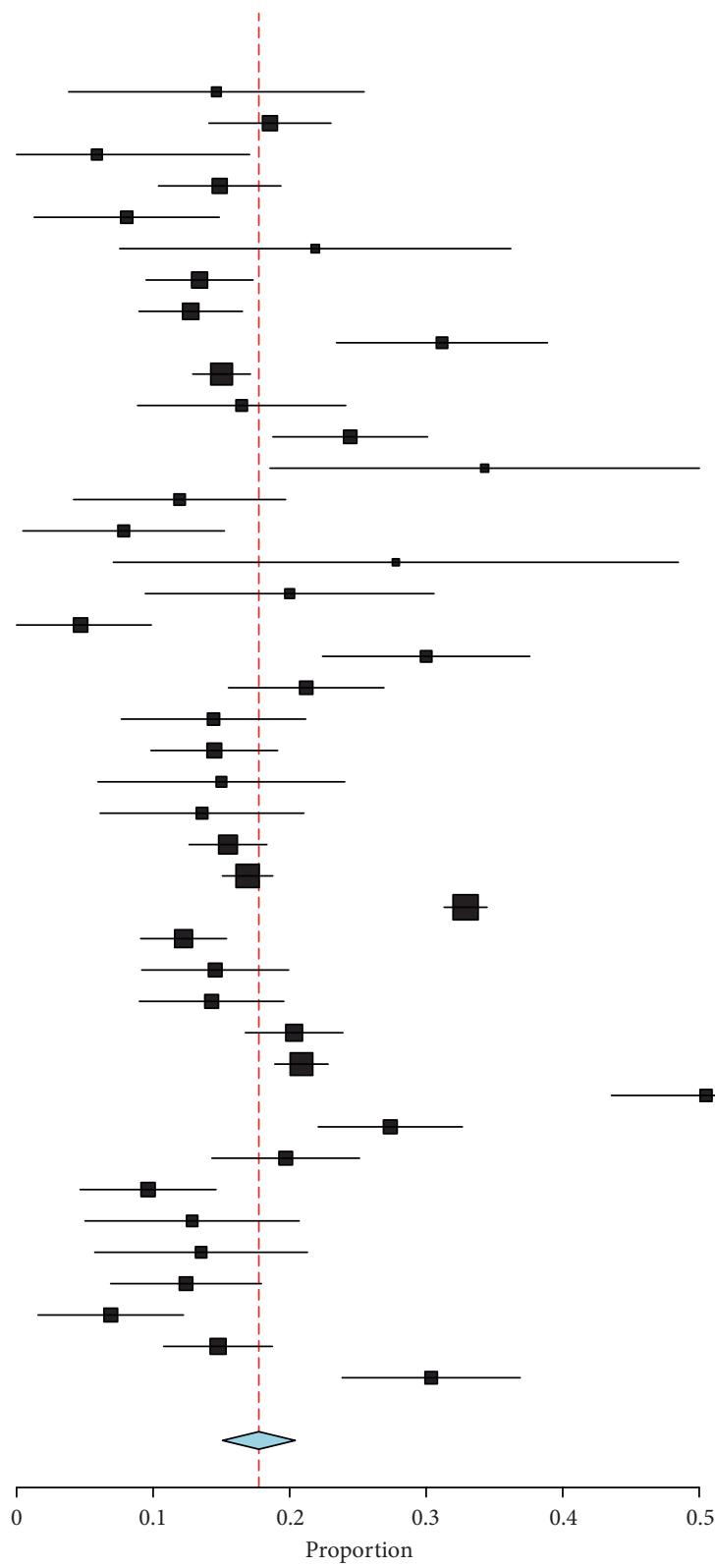

(a)

Figure 2: Continued. 


\begin{tabular}{|c|c|}
\hline Studies & Estimate (95\% C.I.) \\
\hline Youbin Liu 2020 & $0.186(0.141,0.230)$ \\
\hline Jie Li 2020 & $0.059(0.000,0.171)$ \\
\hline Guyi Wang 2020 & $0.149(0.104,0.194)$ \\
\hline Xiaowei Xu 2020 & $0.081(0.013,0.148)$ \\
\hline Wanbo Zhu 2020 & $0.219(0.076,0.362)$ \\
\hline Xu Chen 2020 & $0.134(0.095,0.173)$ \\
\hline Qingxian Cai 2020 & $0.128(0.090,0.165)$ \\
\hline Guoqing Qian 2020 & $0.165(0.089,0.241)$ \\
\hline Yu Lei 2020 & $0.119(0.042,0.197)$ \\
\hline Lei Liu 2020 & $0.078(0.005,0.152)$ \\
\hline Lei Wang 2020 & $0.278(0.071,0.485)$ \\
\hline Penghui Yang 2020 & $0.200(0.094,0.306)$ \\
\hline Min Cao 2020 & $0.212(0.155,0.269)$ \\
\hline Chengfeng Qiu 2020 & $0.144(0.077,0.212)$ \\
\hline Rui Huang 2020 & $0.145(0.098,0.191)$ \\
\hline Zhijun Xie 2020 & $0.150(0.060,0.240)$ \\
\hline Songqiao Liu 2020 & $0.155(0.126,0.183)$ \\
\hline Juan Meng 2020 & $0.122(0.091,0.154)$ \\
\hline Sijiao Wang 2020 & $0.145(0.092,0.199)$ \\
\hline Shijiao Yan 2020 & $0.143(0.090,0.196)$ \\
\hline Yu Shi 2020 & $0.203(0.168,0.239)$ \\
\hline Suxin Wan 2020 & $0.096(0.047,0.146)$ \\
\hline Zhongbao Zuo 2020 & $0.129(0.050,0.207)$ \\
\hline Wei Chen 2020 & $0.135(0.057,0.213)$ \\
\hline Jiaxi Chen 2020 & $0.124(0.069,0.179)$ \\
\hline Wentao Xu 2020 & $0.069(0.016,0.122)$ \\
\hline
\end{tabular}

Overall $\left(I^{\wedge} 2=0 \%, P=0.004\right)$

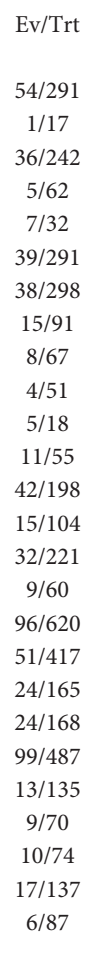

$670 / 4458$

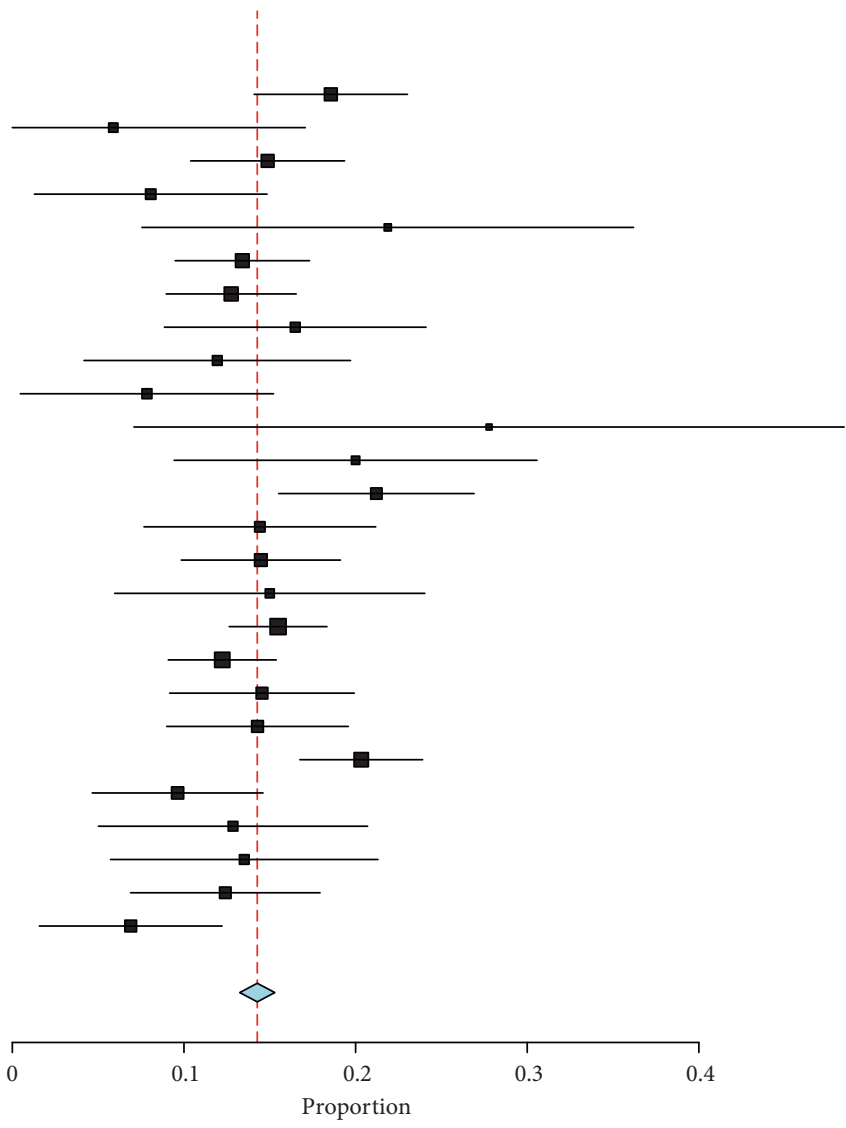

(b)

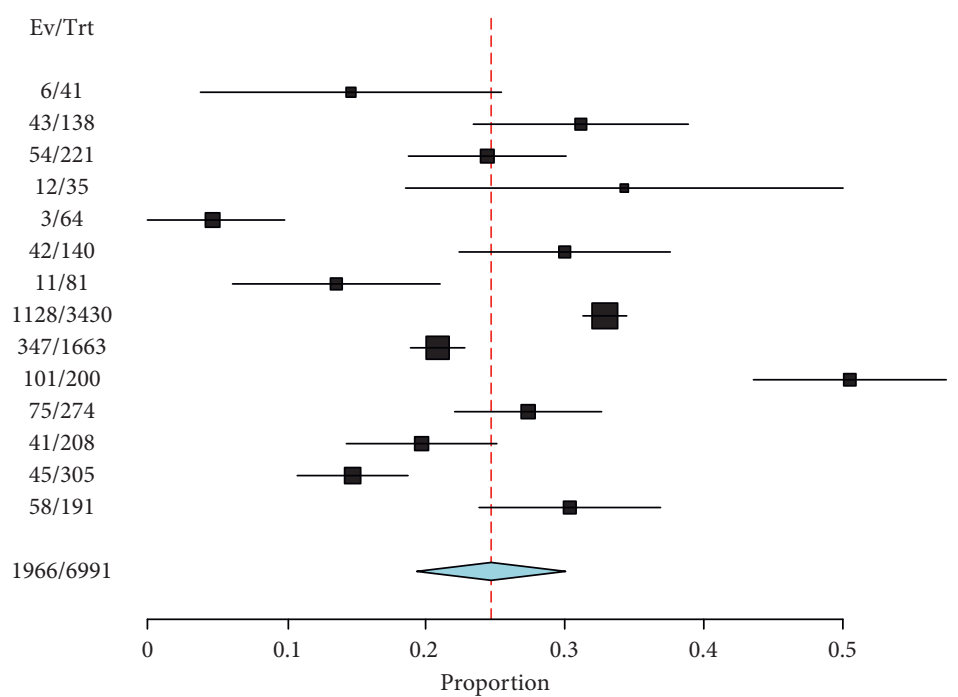

(c)

FIGURE 2: Meta-analysis for the proportion of hypertension in COVID-19 cases. Weights were calculated from binary random-effects model analysis. Values represent the proportion of hypertension in COVID-19 patients and 95\% CI. Heterogeneity analysis was carried out using the Q test among the studies variation ( $I^{2}$ index). (a) The proportion of hypertension in data from all of China. (b) The proportion of hypertension outside Hubei. (c) The proportion of hypertension in Hubei. 


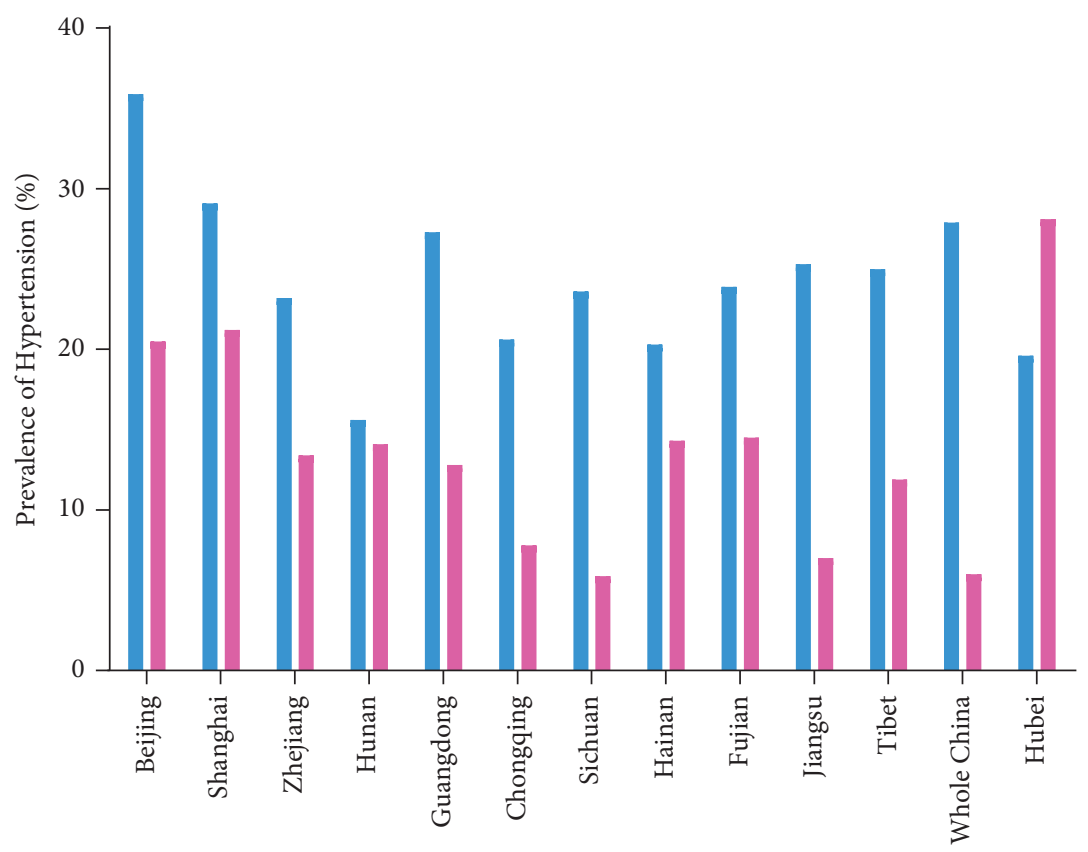

General Population

COVID-19

Figure 3: Comparison of the incidence rates of hypertension in COVID-19 patients with that from the China Hypertension Survey, 2012-2015, in 11 provinces and all of China.

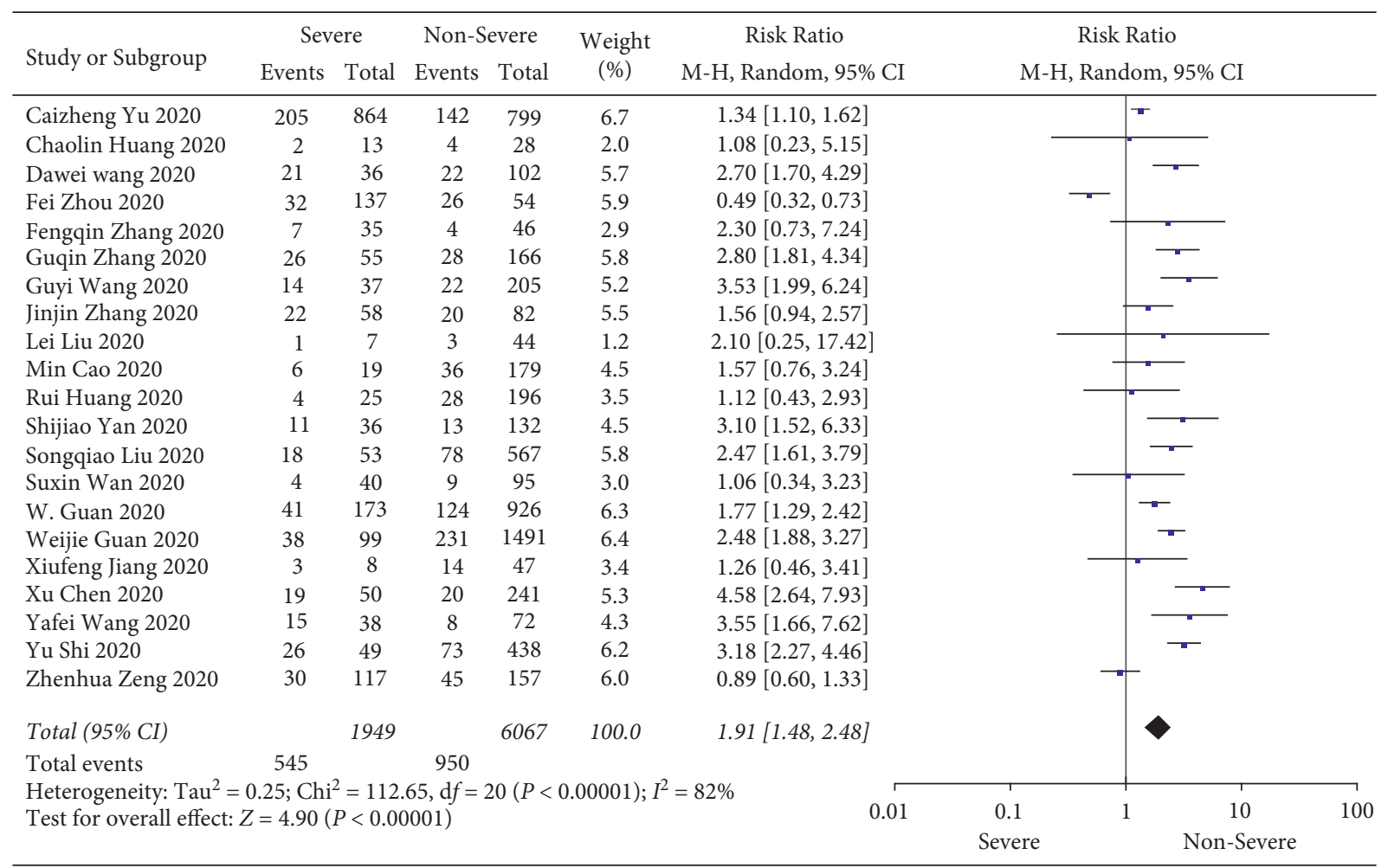

(a)

Figure 4: Continued. 


\begin{tabular}{|c|c|c|c|c|c|c|c|c|c|}
\hline \multirow{2}{*}{ Study or Subgroup } & \multicolumn{2}{|c|}{ Non-Survivors } & \multicolumn{2}{|c|}{ Survivors } & \multirow{2}{*}{$\begin{array}{l}\text { Weight } \\
(\%)\end{array}$} & \multirow{2}{*}{$\begin{array}{c}\text { Risk Ratio } \\
\text { M-H, Random, 95\% CI }\end{array}$} & \multirow{2}{*}{\multicolumn{3}{|c|}{$\begin{array}{c}\text { Risk Ratio } \\
\text { M-H, Random, 95\% CI }\end{array}$}} \\
\hline & Events & Total & Events & Total & & & & & \\
\hline Bo $\mathrm{Hu} 2020$ & 8 & 16 & 10 & 34 & 9.0 & $1.70[0.83,3.48]$ & & $\because$ & \\
\hline Fei Zhou & 26 & 54 & 32 & 137 & 11.7 & $2.06[1.37,3.11]$ & & $\rightarrow$ & \\
\hline Huisi He 2020 & 25 & 42 & 31 & 52 & 12.3 & $1.00[0.71,1.40]$ & & 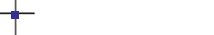 & \\
\hline Kun Wang & 10 & 22 & 35 & 283 & 10.4 & $3.68[2.11,6.39]$ & & - & \\
\hline Lin Fu 2020 & 22 & 34 & 79 & 166 & 12.6 & $1.36[1.01,1.83]$ & & - & \\
\hline Weijie Guan 2020 & 28 & 50 & 241 & 1540 & 12.8 & $3.58[2.73,4.70]$ & & - & \\
\hline Xingwei He 2020 & 12 & 26 & 12 & 28 & 10.0 & $1.08[0.59,1.95]$ & & - & \\
\hline Yimei Yin 2020 & 27 & 52 & 22 & 60 & 11.6 & $1.42[0.93,2.16]$ & & $=$ & \\
\hline Zhenhua Zeng 2020 & 7 & 21 & 68 & 253 & 9.6 & $1.24[0.66,2.35]$ & & - & \\
\hline Total (95\% CI) & & 317 & & 2553 & 100.0 & $1.71[1.19,2.46]$ & & & \\
\hline Total events & 165 & & 530 & & & & & & \\
\hline \multicolumn{7}{|c|}{$\begin{array}{l}\text { Heterogeneity: } \mathrm{Tau}^{2}=0.25 ; \mathrm{Chi}^{2}=53.11, \mathrm{~d} f=8(P<0.00001) ; I^{2}=85 \% \\
\text { Test for overall effect: } Z=2.91(P=0.004)\end{array}$} & $\begin{array}{c}0.1 \\
\text { Non-Survivors }\end{array}$ & $\begin{array}{c}10 \\
\text { Survivors }\end{array}$ & 100 \\
\hline
\end{tabular}

(b)

FIgURE 4: (a) Forest plots depict the comparison of the incidences of hypertension in severe/ICU and nonsevere/ICU patients. (b) Forest plots depict the comparison of the incidences of hypertension in nonsurvivors and survivors. Forest plots depict the comparison of the incidences of cardiac injury in ICU/severe and non-ICU/severe patients.

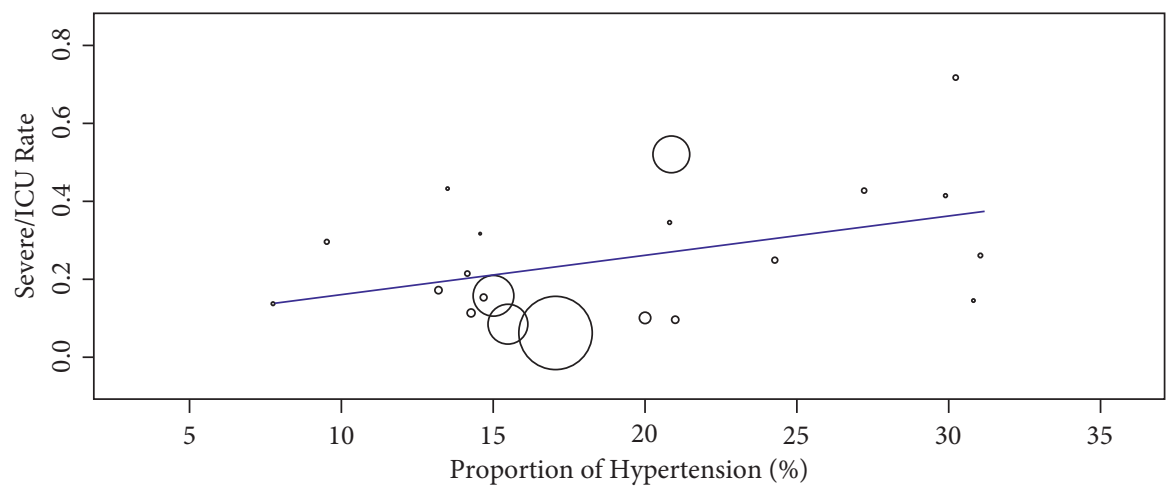

(a)

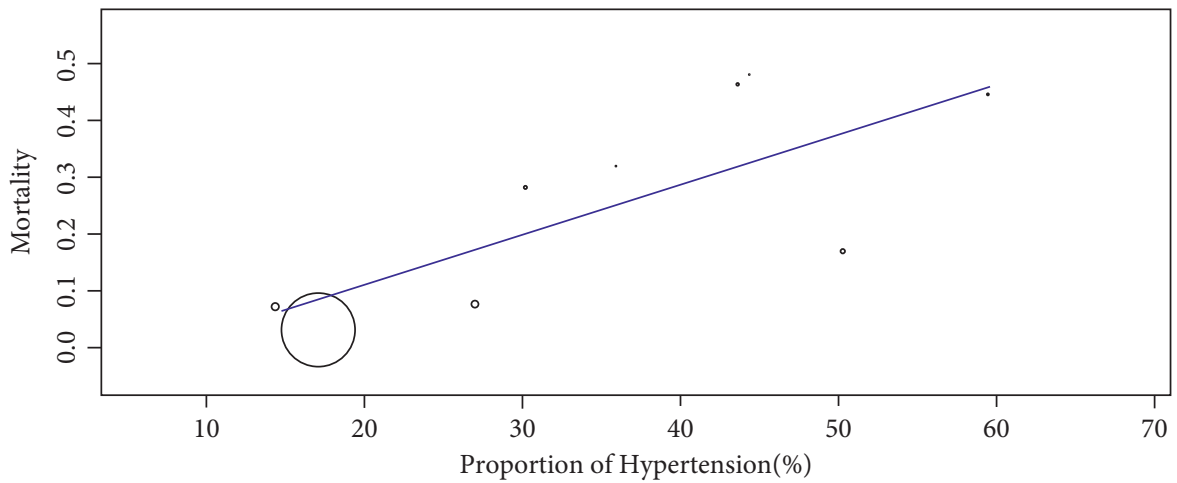

(b)

FIGURE 5: (a) Bubble plots for the association of hypertension with severe/ICU rates in COVID-19 cases. (b) Bubble plots for the association of hypertension with mortality in COVID-19 cases.

antihypertensive drugs recommended in current guidelines. Although ACE is not directly inhibited by ACE inhibitors, ACE2 is affected by chronic treatment with these drugs, which leads to an increase in ACE2 expression in several tissues [44]. Interestingly, this feature is also shared by another drug, angiotensin receptor-1 blocker (ARB).
According to the circulating level of the ACE2 product angiotensin 1-7, long-term administration can also increase the expression level and activity of ACE2 [45]. The use of ACEIs/ARBs in COVID-19 patients with hypertension has caused great controversy. We performed a meta-analysis on the application of ACEIs/ARBs in patients with COVID-19 


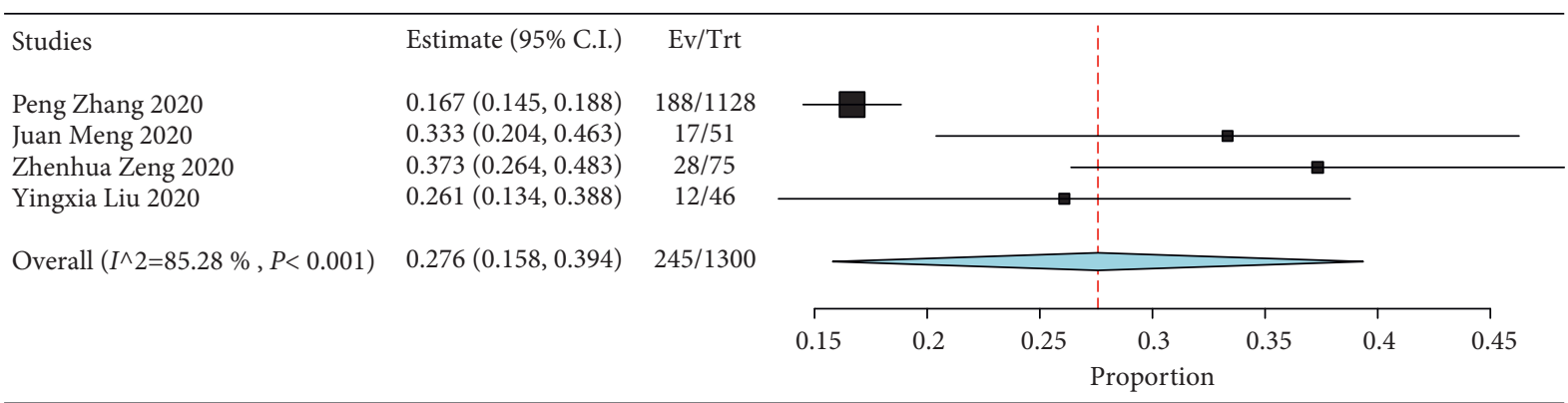

(a)

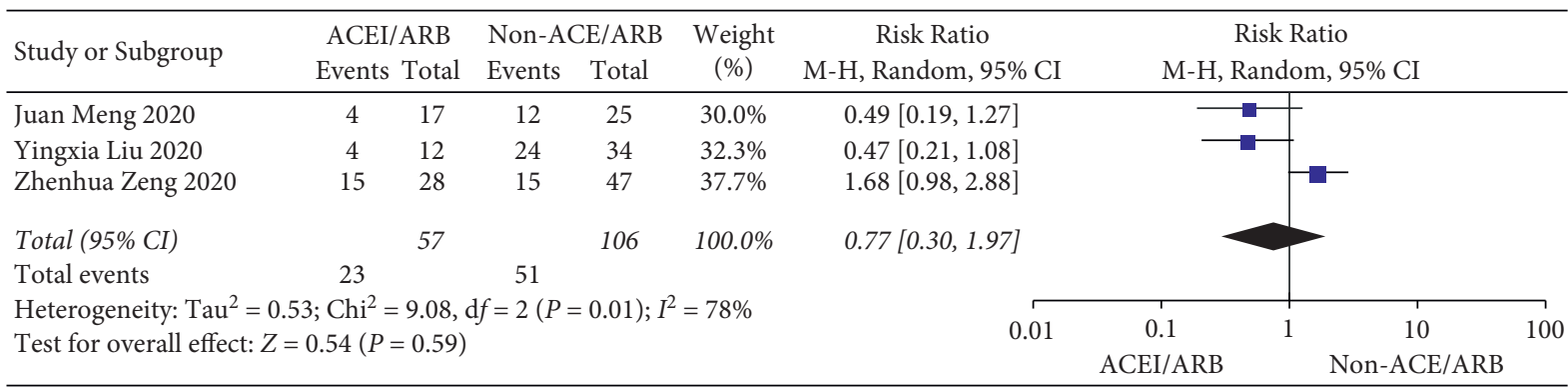

(b)

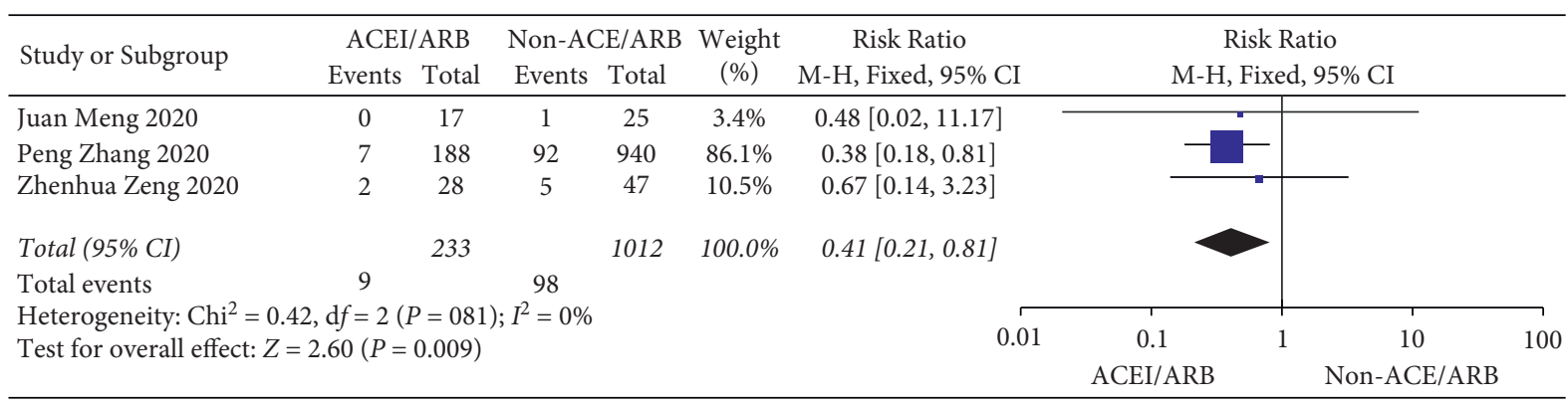

(c)

FIGURE 6: (a) Prevalence of usage of ACEIs/ARBs in COVID-19 patients with hypertension. (b) Comparison of mortality in ACEI/ARB and non-ACEI/ARB patients. (c) Comparison of the incidences of the severe/ICU rate in ACEI/ARB and non-ACEI/ARB patients.

and hypertension and found that $27.6 \%(95 \% \mathrm{CI}$ : $15.8-39.4 \%)$ of patients received ACEI/ARB therapy. The proportion of nonsurvivors or severe patients with hypertension and COVID-19 treated with ACEIs/ARBs was lower than that of survivors or nonsevere patients. The use of ACEIs/ARBs upregulated the expression of ACE2, which might increase susceptibility to COVID-19 and in turn reduce the severity of the disease. In Zhang's study, among hospitalized COVID-19 patients with hypertension, the use of ACEIs/ARBs was associated with a lower risk of all-cause mortality. The upregulation of ACE2 induced by long-term intake of AT1R and ACE inhibitors may play a protective role through the following two mechanisms, first, by blocking the increase of angiotensin 1-7 and, second, by reducing the production of angiotensin II [46].

There were several limitations in the present study. First, there was a difference in the proportion of hypertension among the included cohorts, which may account for the heterogeneity. Occasionally, a small number of samples were reused, which might lead to bias; however, they cannot be excluded crudely in order to avoid bias caused by incomplete data inclusion. Second, the differences in sample size and study design in different studies may be one of the reasons for the heterogeneity. Third, in different studies, severe patients are defined according to different criteria. To simplify the conclusions and improve readability, we combined the severe patients and ICU patients into one category for analysis. In addition, confounding factors (such as sex, smoking, drinking, and history of other comorbidities) in studies may have impacted the outcomes of the patients.

\section{Data Availability}

The data supporting the findings of this study are included within the article.

\section{Additional Points}

Notwithstanding these limitations, our pooled analysis showed that patients with hypertension might be less susceptible to SARS-CoV-2. However, patients with previous 
hypertension may be at increased risk of developing worse clinical outcomes and even death. ACEIs/ARBs can control the blood pressure of COVID-19 patients with hypertension, which may improve the prognosis and reduce mortality.

\section{Conflicts of Interest}

The authors declare that they have no conflicts of interest.

\section{Authors' Contributions}

Bo Li and Lu Zeng contributed equally to this work.

\section{Acknowledgments}

The authors received subsequent financial support for the research, authorship, and/or publication of this article from the China Postdoctoral Science Foundation (No. 2020M682119), Natural Science Foundation of Shandong Province (No. ZR2020MH026), Postdoctoral Innovation Program of Shandong Province (No. 202102041), and Clinical Medicine Science and Technology Innovation Program of Jinan (No. 202019045).

\section{References}

[1] D. Wang, B. Hu, C. Hu et al., "Clinical characteristics of 138 hospitalized patients with 2019 novel coronavirus-infected pneumonia in Wuhan, China," Jama, vol. 323, no. 11, pp. 1061-1069, 2020.

[2] J. J. Zhang, X. Dong, Y. Y. Cao et al., "Clinical characteristics of 140 patients infected with SARS-CoV-2 in Wuhan, China," Allergy, vol. 75, pp. 1730-1741, 2020.

[3] H. Fan, L. Zhang, B. Huang et al., Retrospective Analysis of Clinical Features in 101 Death Cases with COVID-19, medRxiv, 2020.

[4] T. Yao, Y. Gao, Q. Cui et al., "Clinical characteristics of 55 cases of deaths with COVID-19 pneumonia in wuhan, China: retrospective case series," BMC Infectious Diseases, vol. 20, 2020.

[5] B. Zhang, X. Zhou, Y. Qiu et al., "Clinical characteristics of 82 death cases with COVID-19," PLoS One, vol. 15, no. 7, Article ID e0235458, 2020.

[6] Y.-Y. Zheng, Y.-T. Ma, J.-Y. Zhang, and X. Xie, "COVID-19 and the cardiovascular system," Nature Reviews Cardiology, vol. 17, pp. 1-2, 2020.

[7] A. J. Danser, M. Epstein, and D. Batlle, "Renin-angiotensin system blockers and the COVID-19 pandemic: at present there is no evidence to abandon renin-angiotensin system blockers," Hypertension, vol. 120, Article ID 15082, 2020.

[8] B. Li, J. Yang, F. Zhao et al., "Prevalence and impact of cardiovascular metabolic diseases on COVID-19 in China," Clinical Research in Cardiology: Official Journal of the German Cardiac Society, vol. 109, 2020.

[9] W.-j Guan, Z.-y Ni, Y. Hu et al., Clinical Characteristics of 2019 Novel Coronavirus Infection in China, medRxiv, 2020.

[10] C. Novel, "The epidemiological characteristics of an outbreak of 2019 novel coronavirus diseases (COVID-19) in China," Zhonghua Liuxingbingxue Zazhi, vol. 41, no. 2, p. 145, 2020.

[11] Z. Wu and J. M. McGoogan, "Characteristics of and important lessons from the coronavirus disease 2019 (COVID19) outbreak in China: summary of a report of 72314 cases from the Chinese Center for Disease Control and Prevention," Jama, vol. 323, pp. 1239-1242, 2020.

[12] L. Y. Ma, W. W. Chen, R. L. Gao et al., "China cardiovascular diseases report 2018: an updated summary," Journal of geriatric cardiology, vol. 17, no. 1, pp. 1-8, 2020.

[13] L. Fang, G. Karakiulakis, and M. Roth, "Are patients with hypertension and diabetes mellitus at increased risk for COVID-19 infection?" The Lancet. Respiratory Medicine, vol. 8, 2020.

[14] J. Sunden-Cullberg, "Chronic use of angiotensin-converting enzyme inhibitors and angiotensin II receptor blockers is high among intensive care unit patients with non-COVID-19 sepsis but carry a moderately increased risk of death," $\mathrm{Hy}$ pertension, vol. 75, 2020.

[15] P. Zhang, L. Zhu, J. Cai et al., "Association of inpatient use of angiotensin converting enzyme inhibitors and angiotensin II receptor blockers with mortality among patients with hypertension hospitalized with COVID-19," Circulation Research, vol. 126, pp. 1671-1681, 2020.

[16] D. Moher, A. Liberati, J. Tetzlaff, and D. G. Altman, "Preferred reporting items for systematic reviews and meta-analyses: the PRISMA statement," Annals of Internal Medicine, vol. 151, no. 4, pp. 264-269, 2009.

[17] S. Greenland, "Quantitative methods in the review of epidemiologic literature," Epidemiologic Reviews, vol. 9, no. 1, pp. 1-30, 1987.

[18] R. DerSimonian and N. Laird, "Meta-analysis in clinical trials Control," Clinical Trials, vol. 177, p. 10, 1986.

[19] Z. Wang, Z. Chen, L. Zhang et al., "Status of hypertension in China: results from the China hypertension Survey, 2012-2015," Circulation, vol. 137, no. 22, pp. 2344-2356, 2018.

[20] P. Yang, Y. Ding, Z. Xu et al., Epidemiological and Clinical Features of COVID-19 Patients with and without Pneumonia in Beijing, China, medRxiv, 2020.

[21] W. Zhao, S. Yu, X. Zha, W. Ning, Li Dongzeng, and Li Aixin, "Clinical characteristics and durations of hospitalized patients with COVID-19 in Beijing: a retrospective cohort study," Cardiovascular Innovations and Applications, vol. 6, 2020.

[22] M. Cao, D. Zhang, Y. Wang et al., Clinical Features of Patients Infected with the 2019 Novel Coronavirus (COVID-19) in Shanghai, China, medRxiv, 2020.

[23] X.-W. Xu, X.-X. Wu, X.-G. Jiang et al., "Clinical findings in a group of patients infected with the 2019 novel coronavirus (SARS-Cov-2) outside of Wuhan, China: retrospective case series," BMJ, vol. 368, 2020.

[24] G.-Q. Qian, N.-B. Yang, F. Ding et al., "Epidemiologic and clinical characteristics of 91 hospitalized patients with COVID-19 in Zhejiang, China: a retrospective, multi-centre case series," QJM: International Journal of Medicine, vol. 113, 2020.

[25] J. Chen, H. Zhu, D. Wang et al., Clinical Features of Stool SARS-CoV-2 RNA Positive in 137 COVID-19 Patients in Taizhou, China, medRxiv, 2020.

[26] X. Chen, F. Zheng, Y. Qing et al., Epidemiological and Clinical Features of 291 Cases with Coronavirus Disease 2019 in Areas Adjacent to Hubei, China: A Double-Centre Observational Study, medRxiv, 2020.

[27] G. Wang, C. Wu, Q. Zhang et al., Epidemiological and Clinical Features of Corona Virus Disease 2019 (COVID-19) in Changsha, China, 2020.

[28] C. Qiu, Q. Xiao, X. Liao et al., "Transmission and clinical characteristics of coronavirus disease 2019 in 104 outside-Wuhan patients, China," Journal of Medical Virology, vol. 92, 2020.

[29] Q. Cai, D. Huang, P. Ou et al., "COVID-19 in a designated infectious diseases hospital outside Hubei province, China," Allergy, vol. 75, 2020. 
[30] G. Jian-ya, Clinical Characteristics of 51 Patients Discharged from Hospital with COVID-19 in Chongqing, China, medRxiv, 2020.

[31] S. Zhang, H. Li, S. Huang, W. You, and H. Sun, "High-resolution computed tomography features of 17 cases of coronavirus disease 2019 in Sichuan province, China," European Respiratory Journal, vol. 55, no. 4, 2020.

[32] S. Yan, X. Song, F. Lin et al., Clinical Characteristics of Coronavirus Disease 2019 in Hainan, China, medRxiv, 2020.

[33] S. Wang, Z. Chen, Y. Lin et al., Epidemical and Clinical Characteristics of 165 Patients Infected with SARS-CoV-2 in Fujian Province, China, 2020.

[34] W. Xu, S. Qu, M. Xing et al., Epidemiologic Features and Clinical Findings of COVID-19-Infected Patients in Suzhou, 2020.

[35] Y. Lei, X. Huang, B. SiLang, Y. P. Lan, J. Lu, and F. Zeng, Clinical Features of Imported Cases of Coronavirus Disease 2019 in Tibetan Patients in the Plateau Area, medRxiv, 2020.

[36] C. Vickers, P. Hales, V. Kaushik et al., "Hydrolysis of biological peptides by human angiotensin-converting enzyme-related carboxypeptidase," Journal of Biological Chemistry, vol. 277, no. 17, pp. 14838-14843, 2002.

[37] M. A. Crackower, R. Sarao, G. Y. Oudit et al., "Angiotensinconverting enzyme 2 is an essential regulator of heart function," Nature, vol. 417, no. 6891, pp. 822-828, 2002.

[38] S. Wakahara, T. Konoshita, S. Mizuno et al., "Synergistic expression of angiotensin-converting enzyme (ACE) and ACE2 in human renal tissue and confounding effects of hypertension on the ACE to ACE2 ratio," Endocrinology, vol. 148, no. 5, pp. 2453-2457, 2007.

[39] K. Kuba, Y. Imai, and J. Penninger, "Angiotensin-converting enzyme 2 in lung diseases," Current Opinion in Pharmacology, vol. 6, no. 3, pp. 271-276, 2006.

[40] P. Balakumar and G. Jagadeesh, "A century old renin-angiotensin system still grows with endless possibilities: AT1 receptor signalling cascades in cardiovascular physiopathology," Cellular Signalling, vol. 26, no. 10, pp. 2147-2160, 2014.

[41] B. Rodriguez-Iturbe, H. Pons, and R. J. Johnson, "Role of the immune system in hypertension," Physiological Reviews, vol. 97, no. 3, pp. 1127-1164, 2017.

[42] Y. Zhang, P. Murugesan, K. Huang, and H. Cai, "NADPH oxidases and oxidase crosstalk in cardiovascular diseases: novel therapeutic targets," Nature Reviews Cardiology, vol. 17, pp. 1-25, 2019.

[43] A. Kaparianos and E. Argyropoulou, "Local renin-angiotensin II systems, angiotensin-converting enzyme and its homologue ACE2: their potential role in the pathogenesis of chronic obstructive pulmonary diseases, pulmonary hypertension and acute respiratory distress syndrome," Current Medicinal Chemistry, vol. 18, no. 23, pp. 3506-3515, 2011.

[44] L. S. Zisman, G. E. Meixell, M. R. Bristow, and C. C. Canver, "Angioten - sin-(1-7) formation in the intact human heart: in vivo dependenceon angiotensin II as substrate," Circulation, vol. 108, no. 14, pp. 1679-1681, 2003.

[45] C. M. Ferrario, J. Jessup, M. C. Chappell et al., "Efect of angiotensin-converting enzyme inhibition and angiotensin II receptor blockers on cardiac angiotensin-converting enzyme 2," Circulation, vol. 111, no. 20, pp. 2605-2610, 2005.

[46] A. Albini, D. M. Noonan, G. Pelosi, G. Di Guardo, and M. Lombardo, "The sars-cov-2 receptor, ace-2, is expressed on many different cell types: implications for ace-inhibitor- and angiotensin ii receptor blocker-based antihypertensive therapies-reply," Internal and Emergency Medicine, vol. 15, no. 8, 2020 . 